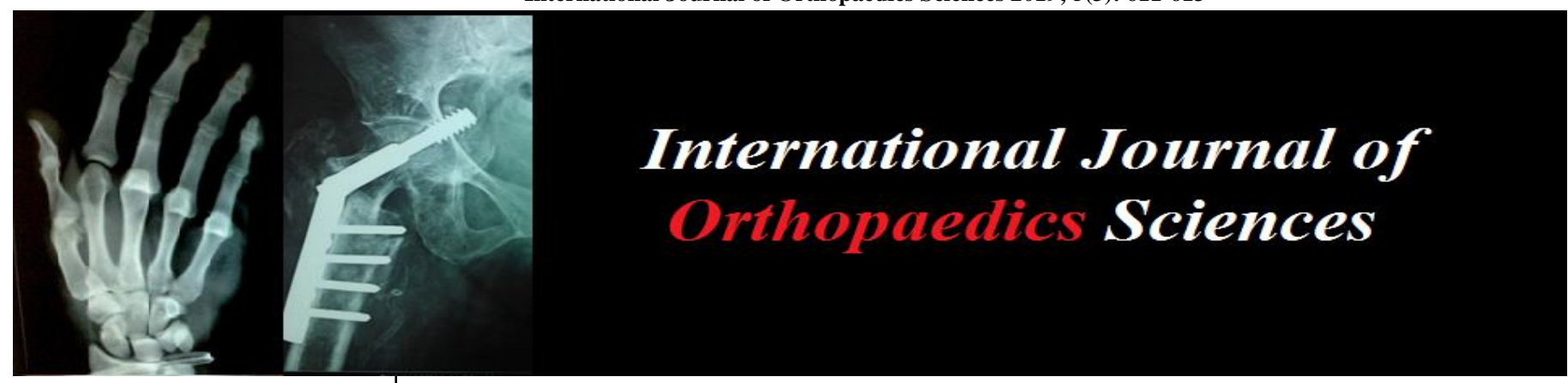

ISSN: $2395-1958$

IJOS 2019; 5(3): 611-613

(C) 2019 IJOS

www.orthopaper.com

Received: 04-05-2019

Accepted: 06-06-2019

Dr. Saurabh Kumar

U.C.M.S \& G.T.B Hospital, Delhi, India

Dr. Deepankar Verma

U.C.M.S \& G.T.B Hospital, Delhi, India

Dr. Ish Kumar Dhammi U.C.M.S \& G.T.B Hospital, Delhi, India

Dr. Rajnand Kumar U.C.M.S \& G.T.B Hospital, Delhi, India

Dr. Samarth Mittal A.I.I.M.S, Rishikesh, Uttarakhand, India

Correspondence

Dr. Deepankar Verma U.C.M.S \& G.T.B Hospital, Delhi, India

\section{Transverse sacral fracture-dislocation with anterior displacement in 13s year old female: A case report}

\author{
Dr. Saurabh Kumar, Dr. Deepankar Verma, Dr. Ish Kumar Dhammi, \\ Dr. Rajnand Kumar and Dr. Samarth Mittal
}

DOI: https://doi.org/10.22271/ortho.2019.v5.i3k.1597

\section{Abstract}

Introduction: Sacrum fractures are rare pathologies seen after spinal traumas. The incidence of a sacral fracture after trauma is $0.6 \%$ in childhood.

Case presentation: A 13-year-old girl was admitted to our emergency room after having suffered a fall. Radiological tests revealed S1 S2 fracture dislocation. Appropriate load distribution through a spinopelvic fixation as well as neural decompression were performed together with an S1-S2 partial laminectomy. Post operatively iliac screws started impinging so all the screws were removed 3 months post operatively. At one year follow - up patient is walking pain free without any neurological deficit. Conclusion: It is very difficult to make a generalization for treatment of sacral fracture dislocation due to small number of patients. Each patient should be individualized and lumbosacroiliac instability should be treated.

Keywords: Transverse sacral fracture-dislocation, anterior displacement, 13 year old female

\section{Introduction}

Sacral fracture dislocation especially transverse fracture with anterior displacement is an uncommon injury. They are classified into longitudinal and transverse fracture. Transverse fracture constitutes $3 \%$ to $5 \%$ with most of fractures (95\% to $97 \%$ ) being longitudinal fractures Transverse fractures are usually observed following a fall from height, thus it is also named "suicide jumper's fracture" [1]. Neurological deficit involving sacral roots are common in transverse fractures (96\% to $100 \%$ ). We present such a case in a child who was managed successfully by spino pelvic fixation, attempt of reduction, partial limited laminectomy at S1 S2.

\section{Case Report}

A 13-year-old female who fell down from $10-12 \mathrm{ft}$ height and presented in our emergency department with pain in abdomen, lower back pain, inability to pass urine. Patient was haemodynamically unstable at presentation with pulse rate $112 / \mathrm{min}$, B.P $80 / 40 \mathrm{~mm} \mathrm{Hg}$, RR $24 / \mathrm{min}$. She was resuscitated with administration of adequate fluids, colloids and blood through two large bore cannulation and oxygen inhalation. USG FAST was done which came to be positive. On further radiological investigations CECT abdomen showed mild haemoperitoneum and mild retroperitoneal hematoma on right side. CT spine showed transverse S1 S2 fracture dislocation.(Fig1) The patient was initially admitted in surgery department and was kept under observation and treated conservatively from their side and subsequently transferred to orthopaedics department after $72 \mathrm{hrs}$ for managing sacral fracture dislocation. The patient was hemodynamically stable when presented in orthopaedics department with pulse rate 80/min and B.P 110/80 $\mathrm{mm} \mathrm{Hg}$. she had tenderness on lumbosacral area, with bony protrusion at S1S2. Pelvic compression distraction test was positive, active straight leg raising test was negative. There was no limb length discrepancy. On examination she had superficial abrasions present over lower back and bruises present over bilateral flanks. On neurological examination exact power could not be assessed as she was having severe pain and spasm, no sensory deficit with bladder, bowel involvement. Radiographs of LS spine AP and lateral views, pelvis with both hip joints and pelvis inlet/outlet views were obtained. 
It showed fracture of $\mathrm{S} 1$ and $\mathrm{S} 2$ vertebra with dislocation of S1 over S2 (fig2). Bilateral sacroiliac joint were intact with no other fracture in any other bone. Surgical intervention was undertaken on day 11 post-trauma after pre anaesthetic fitness. A dorsal midline approach was used with the patient in prone position. The fracture dislocation was reduced and stabilized by inserting pedicle screws at both L4 and L5, and bilateral iliac pedicle screws inserted inferior to posterior iliac spine, aimed towards the acetabular dome (spino- pelvic fixation (Fig3). There was CSF leak from small rent which was managed conservatively Postoperative the iliac screws started impinging (fig4) on the skin so the screws were removed at three months postoperatively and radiographs are depicted in fig5. 12 months post operative patient is walking pain free without any neurological deficit (fig6)

\section{Discussion}

Transverse sacral fractures are rare and constitute less than $1 \%$ of all spinal fractures. Whilst the majority are longitudinal fractures, only $3 \%$ to $5 \%$ are transverse fractures ${ }^{[2]}$. As a result of low incidence and the radiological difficulties in visualizing the fracture, accurate diagnosis is often delayed or unrecognized. We detected early as CECT abdomen for haemoperitoneum. In the report of Denis and associates [2], regarding patients with neurological deficit, $49 \%$ of fractures were not associated with neural deficit. In patients without neurological deficit, only $5 \%$ were diagnosed on initial hospitalization. In another series by Roy-Camille and colleagues, $54 \%$ of patients had the diagnosis delayed by one to 18 months ${ }^{[1]}$.

Conventional radiographs are often inadequate for clear visualization of the fracture as two iliac bones cover the sacrum. In patients with a history of high-energy trauma and clinical signs suggestive of lumbosacral injury, additional radiographs, such as lateral sacral, pelvic inlet and outlet, and Ferguson's view may improve the visualization of a sacrum fracture. CT scans with $3 \mathrm{D}$ reconstruction are very useful to demonstrate the full extent of this injury. The role

of MRI is to determine spinal cord or nerve root injuries when neurological deficit is present and planning surgery. In this case, the presence of bladder incontinence alerted us to a possible lumbosacral injury, and CT scan was performed to confirm the diagnosis. Denis et al. proposed a classification system based on anatomic location of fractures as follows: zone I involves the alar region; zone II fracture occurs at the sacral foraminal area; and zone III involves the central canal. Transverse fractures are considered zone III injuries, as there is involvement of the spinal canal ${ }^{[2]}$. Roy-Camille further classified transverse sacral fracture into three types ${ }^{[1]}$, and a new type of fracture was added later to this classification paradigm by Strange-Vognsen: type I injury is angulated but not translated; type II is angulated and translated; type III shows complete translational displacement of the cephalad and caudal parts of the sacrum; and type IV is segmentally comminuted as a result of axial compression. By definition, this case represents a type III Roy-Camille fracture. Neurological disturbances are quite common in transverse sacral fractures. Denis et al. report neurological deficit in $56 \%$ of zone III injuries ${ }^{[2]}$. In some larger series of transverse sacral fracture, $96 \%$ to $100 \%$ of patients had some neurological deficit 1,3, mainly in the form of bowel and bladder dysfunction and saddle anaesthesia, similar to what we found in this patient.

Management of these injuries remains controversial. Various treatments have been reported in the literature. These include conservative management, initial conservative treatment followed by surgical treatment after failure of conservative treatment, and primary surgical treatment. Conservative treatment includes bed rest with or without traction for 3 to 8 weeks. The proponents of conservative treatment stated that spinal nerves have the ability to progressively restore function although the recovery may be incomplete ${ }^{[4]}$. The drawbacks of non-operative treatment are residual pelvic discomfort, low back pain and neurological deficit. A trial of closed reduction may be attempted using heavy two-pole traction to improve sagittal alignment of the lumbosacral junction. However, due to the unstable nature of this injury, loss of reduction may occur over time and necessitate surgery. Surgical treatment is indicated for significant displacement, neurological deficit, instability or deterioration after nonoperative treatment. Options include decompression with laminectomy, laminectomy and stabilization, or stabilization alone. Various methods of stabilization have been reported, including Harrington, Luque, plating, lumbosacral pedicle screws extending to S2, percutaneous sacroiliac screws, and lumbopelvic constructs. Due to the rarity of the injury, we reviewed the literature before embarking on treatment decision. In this patient, S1/S2 fracture-dislocation with diastases of bilateral sacroiliac joint was the cause of the separation of upper sacral fragments from pelvic and lower sacral segments, giving rise to a condition called "spinopelvic dissociation".

The literature suggests that lumbopelvic fixation is best indicated for spino-pelvic dissociation as it mimics the normal load transfer by unloading the sacral fracture. To our knowledge, Schildhauer et al. ${ }^{[5]}$ reported on the largest series of patients treated successfully using lumbopelvic fixation. They used two pairs of pedicle screws for fixation of the lumbosacral spine, and two points of iliac fixation bilaterally with either iliac screw alone or in combination with iliosacral screws. In his results, sacral fractures healed in all 18 patients without loss of reduction. Average kyphosis improved more than $50 \%$, and $83 \%$ of patients had full or partial recovery of bowel and bladder deficits. Based on the above reasons, we advocated similar surgical method for our patient. Laminectomy was not performed as the neurological deficit was caused by compression at the dislocation site. Although the reduction was limited, neurological recovery was good at follow-up. The patient later underwent removal of the implant as a result of prominence of iliac screw. Schildhauer et al. ${ }^{[5]}$ had also reported this as a hardware complications. Other complications reported were wound-related problems such as infection, hematoma or seroma formation, which were not seen in this patient.

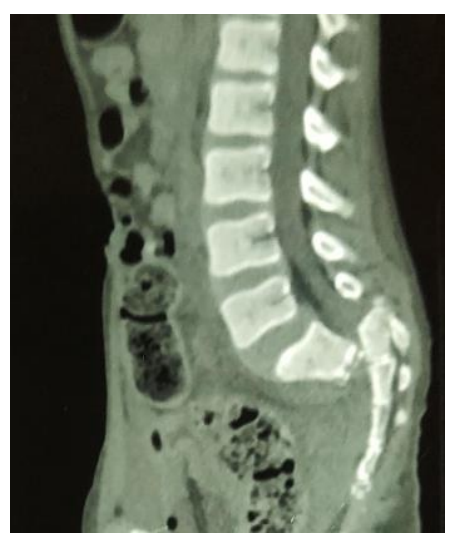

Fig 1: CT film showing S1S2 fracture dislocation 

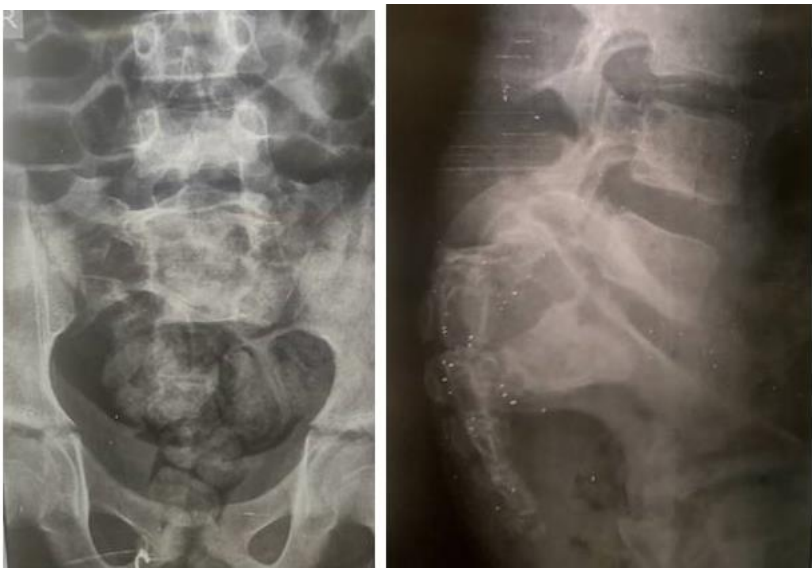

Fig 2: X ray showing S1S2 fracture dislocation
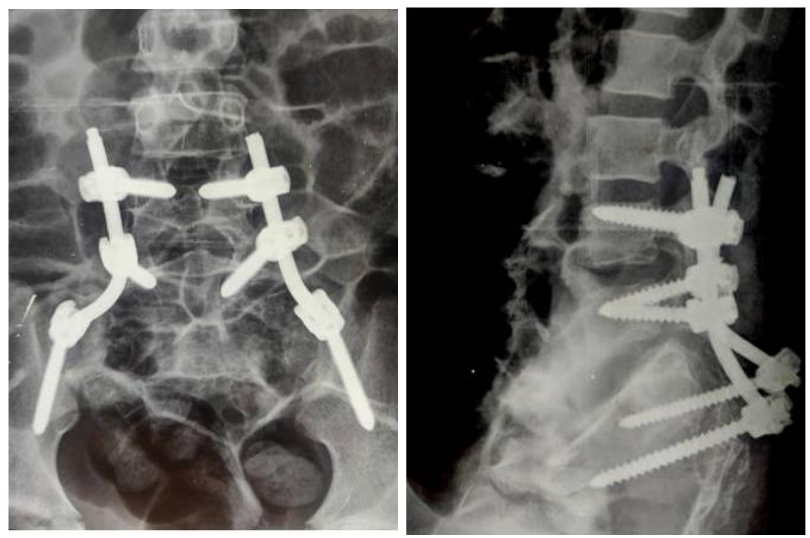

Fig 3: post operative Xray showing spino pelvic fixation

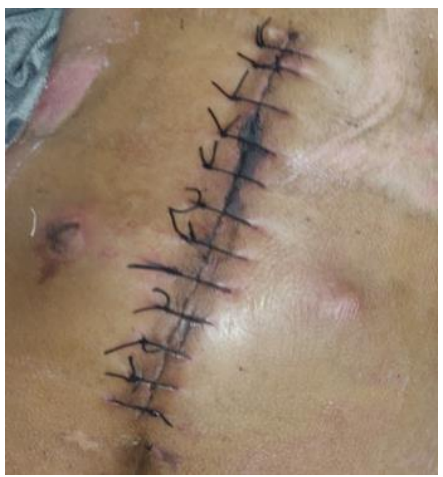

Fig 4: impingement of iliac screws on skin

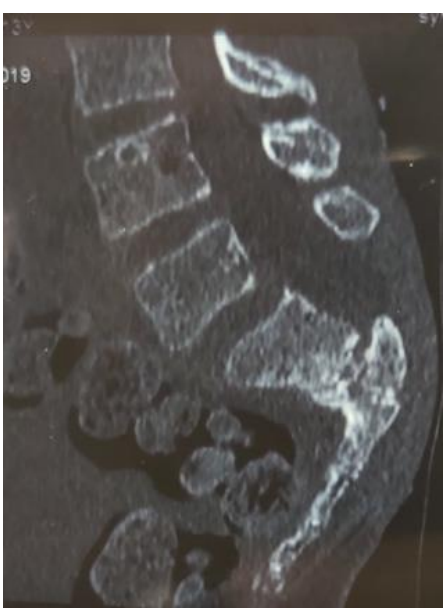

Fig 5: radiograph after screw removal/radiograph at one year follow up
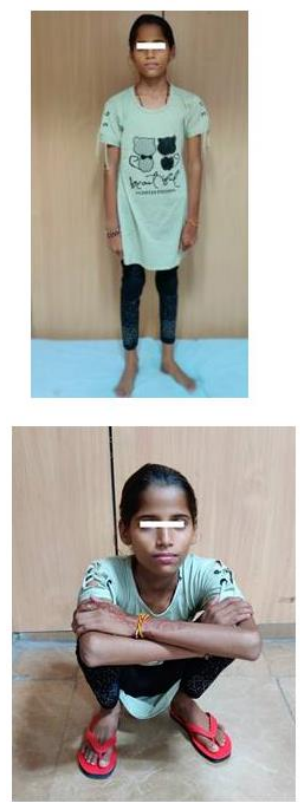
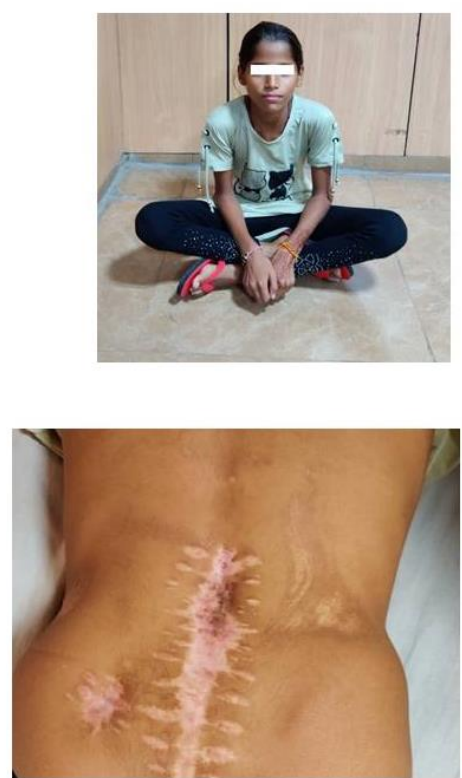

Fig 6: clinical photographs at one year follow-up

\section{Conclusion}

A transverse sacral fracture is an uncommon but severe injury. It is frequently missed and unrecognized, the upper sacrum is usually involved and most patients present with neurological deficit awareness about this injury is worth. Operative treatment is often recommended for significant displacement, neurological deficit or deterioration in condition for early mobilization and sitting.

Competing interests: The author(s) declare that they have no competing interests".

\section{Patient consent: Obtained}

\section{References}

1. Roy Camille R, Saillant G, Gagna G, Mazel C. Transverse fracture of the upper sacrum. Suicidal jumper's fracture. Spine. 1985; 10:838-45.

2. Denis F, Davis S, Comfort T. Sacral fractures: an important problem. Retrospective analysis of 236 cases. Clin Orthop. 1988; 227:67-81.

3. Sabiston CP, Wing PC. Sacral fractures: Classification and neurologic implications. J Trauma. 1986; 26: 1113-5.

4. Phelan ST, Jones DA, Bishay M. Conservative management of transverse fractures of the sacrum with neurological features. A report of four cases. J Bone Joint Surg Br. 1991; 73(6):969-71.

5. Schildhauer TA, Bellabarba C, Nork SE, Barei DP, Chip Routt ML, Chapman JR. Decompression and lumbopelvic fixation for sacral fracture-dislocation with spino-pelvic dissociation. J Orthop Trauma. 2006; 20:447-57. 